should be subject to the same regulations as those operating in such international laboratories. The Committee also feels that if Denmark sticks to common guidelines, it will be able to call on experts in the ESF's 'Liaison committee on recombinant DNA research'-on which it is representedfor advice with specific genetic engineering problems.

The report's recommendations aim at controlling public as well as private research and ensuring that the community has the power to regulate the use of any results from genetic engineering research which may pose hazards to the environment, animals or man. And that power, the report says, should eventually be vested in the Commission for Genetic Engineering when it is set up.

All research and production using genetic engineering will then have to be made known to the Commission and particularly hazardous procedures will not be allowed to go ahead without the Commission's approval. Laboratories will have to be approved by the Directorate of Labour Inspection, or by a public or private institution authorised by the Directorate in collaboration with the Commission. People working with genetic engineering will have to be adequately trained; accurate records will have to be kept of all experiments; accidents will have to be immediately notified to the Commission and laboratories will have to be inspected regularly.

A point which the Registration Committee suggests should be looked into further is the question of whether existing Danish law (the Epidemics Act 1915, the Working Environment Act 1975, the Environment Protection Act 1973) affords a sufficient supervisory authority to inspect laboratories or whether special legislation will be needed. Eventually, however, recommendations from the European Economic Community (EEC) will have to be taken into account on the latter point. As yet no decision has been taken on which central authority the Commission should be placed under, but the Registration Committee suggests that the National Health Service, which supervises other forms of microbiological research, might be the most suitable.

The Registration Committee's next task will be to collaborate with the National Health Service, the Directorate of Labour Inspection and the Agency of Environmental Protection to work out preliminary guidelines for registration, supervision, approval of laboratory facilities and so on. In the meantime it will keep the government and administrative authorities informed of developments abroad.

Sven Godtfredsen

\title{
First Ratan results published
}

THE first results of experiments with Ratan- 600 , the $600 \mathrm{~m}$ radio-telescope of the Soviet Academy of Sciences, have recently been published. The experiments are essentially exploratory and performance trials ranging from solar to extra-galactic observations.

The Ratan-600 represents a new generation of radio-telescopes. Its design parameters are impressive: information capacity $3 \times 10^{12}$ bits, limiting resolution 2 arc sec, accuracy of definition of coordinates $0.01 \mathrm{sec}^{2}$, limiting flux density 0.01 Jansky, limiting brightness temperature $0.03 \mathrm{~K}$, sky coverage $70-80 \%$ with scanning time 100 days. Ultimately it is hoped that Ratan-600 will not only provide direct observational results, but will serve as a basis for experiments estimating the intensity of gravitational waves and confirming general relativity on the basis of the bending of rays in the gravitational field of the sun. Although these sophisticated experiments lie in the future, a number of interesting results have already been obtained.

The high resolution and large collecting area of RATAN-600 permit the observation of solar "pores"-areas of limitingly weak activity which will later develop into sunspots. It also allows the radiogranules, first discovered during the transit of Mercury in 1970 to be monitored in non-eclipse conditions and for several frequencies simultaneously. A programme of joint radio and optical investigation of solar granules has been initiated and preliminary investigations obtained for their magnetic fields.

Likewise, the high resolution made it possible for RATAN-600 to receive radio-emissions from all four of the "Galilean" satellites of Jupiter, in spite of the intense radio-emission from the planet itself. These observations-in December 1976 and January 1977, gave the first results ever for Io, the closest, and Europa, the nearest, of the four. For Ganymede, Callisto and Europa, the results were consistent with thermal emission only, showing no signs of the intense radiation bands observed for Jupiter itself. For Io, however, the result was "totally unexpected"-radio-emission is several times greater than the expected thermal value. It is claimed that this implies that Io possesses a magnetic field and intense radiation bands.

RATAN-600 is aiso participating in two joint Soviet-Australian projects, on quasars and on early-type emission line stars. Signals have been received from quasar $O Q$ 172, the most remote object in the universe (red shift 3.54). Its present programme also includes observations of the galactic centre, multifrequency mapping of radiogalaxies, and the first stages of a sky survey in the centimetre and millimetre ranges, which will take 10 years to complete and should provide a radio equivalent of the Palomar sky survey.

Vera Rich

\section{Bangladesh plans nuclear research centre...}

Bangladesh Atomic Energy Commission, in its efforts to develop peaceful applications of nuclear physics and technology, has drawn up an extensive plan to establish a nuclear research complex in Bangladesh. It will comprise seventeen nuclear and allied projects. A 259-acre site twenty-five miles north of Dacca is already under development. The laboratories are to be integrated to share facilities and also to create an environment conducive to the formation of interdisciplinary teams among the scientists of the Commission.

$\mathrm{Mr}$ H. F. S. Bittencourt, Deputy Director General of the International Atomic Energy Agency, while visiting Bangladesh in connection with the inaugural ceremony of the Institutes of Nuclear Technology, Nuclear Medicine, Electronics, Irradiation and Pest Control, the National Com- puter Centre, the Institute of Space and Atmospheric Research, and other institutes, expressed his satisfaction over the plans and assured all possible help in matters of providing the Commission with a small nuclear reactor. It is expected that the first phase of the work will be completed by 1982 , but Bangladesh still needs a 10 to $20 \mathrm{MW}$ reactor, which the BAEC hopes to get as a donation from some friendly country. Bangladesh, however, is required to obtain a nuclear safeguard clearance before obtaining a nuclear reactor and it is hoped that the IAEA will extend the necessary help.

Two irradiators each of 50,000 Curies for food irradiation and medical projects are to be procured from Canada. The first one which is expected to arrive in June next, will cost about US\$6,000.

M. Kabir 